\title{
Dynamic Electronic Tracking and Escalation to reduce Critical care Transfers (DETECT): the protocol for a stepped wedge mixed method study to explore the clinical effectiveness, clinical utility and cost- effectiveness of an electronic physiological surveillance system for use in children
}

Gerri Sefton ${ }^{1 *}$, Bernie Carter ${ }^{2}$, Steven Lane ${ }^{3}$, Matthew Peak ${ }^{4}$, Ceu Mateus ${ }^{5}$, Jen Preston ${ }^{3}$, Fulya Mehta ${ }^{1}$, Bruce Hollingsworth ${ }^{5}$, Roger Killen ${ }^{6}$ and Enitan D. Carrol ${ }^{3}$

\begin{abstract}
Background: Active monitoring of hospitalised adults, using handheld electronic physiological surveillance systems, is associated with reduced in-patient mortality in the UK. Potential also exists to improve the recognition and response to deterioration in hospitalised children. However, the clinical effectiveness, the clinical utility, and the cost-effectiveness of this technology to reduce paediatric critical deterioration, have not been evaluated in an NHS environment.

Method: This is a non-randomised stepped-wedge prospective mixed methods study. Participants will be in-patients under the age of 18 years, at a tertiary children's hospital. Day-case, neonatal surgery and Paediatric Intensive Care Unit (PICU) patients will be excluded.

The intervention is the implementation of Careflow Vitals and Connect (System C) to document vital signs and sepsis screening. The underpinning age-specific Paediatric Early Warning Score (PEWS) risk model calculates PEWS and provides associated clinical decision support. Real-time data of deterioration risk are immediately visible to the entire clinical team to optimise situation awareness, the chronology of the escalation and response are captured with automated reporting of the organisational safety profile.

Baseline data will be collected prospectively for 1 year preceding the intervention. Following a 3 month implementation period, 1 year of post-intervention data will be collected.

The primary outcome is unplanned transfers to critical care (HDU and/or PICU). The secondary outcomes are critical deterioration events (CDE), the timeliness of critical care transfer, the critical care interventions required, critical care length of stay and outcome.

The clinical effectiveness will be measured by prevalence of CDE per 1000 hospital admissions and per 1000 non-PICU bed days.

(Continued on next page)
\end{abstract}

\footnotetext{
* Correspondence: gerri.sefton@alderhey.nhs.uk

'Alder Hey Children's NHS Foundation Trust, Liverpool L12 2AP, UK

Full list of author information is available at the end of the article
}

C The Author(s). 2019 Open Access This article is distributed under the terms of the Creative Commons Attribution 4.0 International License (http://creativecommons.org/licenses/by/4.0/), which permits unrestricted use, distribution, and reproduction in any medium, provided you give appropriate credit to the original author(s) and the source, provide a link to the Creative Commons license, and indicate if changes were made. The Creative Commons Public Domain Dedication waiver (http://creativecommons.org/publicdomain/zero/1.0/) applies to the data made available in this article, unless otherwise stated. 


\begin{abstract}
(Continued from previous page)
Observation, field notes, e-surveys and focused interviews will be used to establish the clinical utility of the technology to healthcare professionals and the acceptability to in-patient families. The cost-effectiveness will be analysed using Health Related Group costs per day for the critical care and hospital stay for up to 90 days post CDE.
\end{abstract}

Discussion: If the technology is effective at reducing CDE in hospitalised children it could be deployed widely, to reduce morbidity and mortality, and associated costs.

Trial registration: Current Controlled Trials ISRCTN61279068, date of registration 03.06.19, retrospectively registered.

Keywords: Deterioration, Sepsis, VitalPAC ${ }^{\circledast}$, Paediatric early warning (PEWS), E-obs, Careflow vitals and connect

\section{Background}

All-cause child mortality in the UK has not maintained improvement, in line with other European countries. The Confidential Enquiry into Maternal and Child Health deaths (CEMACH) 'Why children die", found that $25 \%$ of in-hospital deaths had identifiable failures in direct care and potentially avoidable factors in $43 \%$ [1, 2]. The factors included failure to recognise and respond effectively to deterioration. Paediatric Early Warning Scores (PEWS) and systems have been advocated as a mechanism to assist healthcare staff to identify deterioration so that care might be augmented to improve outcome. However, the evidence base underpinning their use in clinical practice is limited [1]. In the UK, despite reported use of PEWS by $85 \%$ of hospital treating children [3], there are more than 4000 emergency transfers to Paediatric Intensive Care Units per year from inpatient wards [4], suggesting that those patients deteriorated in hospital. The progression of deterioration to become critical, requiring admission to a high dependency unit (HDU) or the PICU is known to increase the length of hospital stay and the risk of mortality [5-7]. Evolving evidence suggests that PEWS may assist in reducing the severity of illness and the critical interventions required by patients who require unplanned emergency transfers to critical care $[8,9]$.

To date, PEWS research has been hampered by the use of paper-based charting of vital signs. Achieving implementation fidelity of PEWS systems have been challenging, with incomplete monitoring of the specific vital signs that contribute to PEWS reported. Additionally, there has been poor documentation of PEWS escalation, the timeliness of response and the associated clinical outcomes, which makes it difficult to assess if PEWS have been implemented as intended. In contrast, active monitoring of adult patients in hospital using an electronic physiological surveillance system has been in place for more than 10 years [10]. This technology has been associated with the standardisation of vital signs monitoring and has demonstrated a significant reduction in deaths at two large University hospitals in the UK [11]. That work contributed to the development of a National Early Warning Score
(NEWS), a single risk model for identifying signs of early deterioration in adults [12-14].

\section{Why this study is necessary and the expected contribution to the field}

The risk model developed for adults is unsuitable for use in children for a number of reasons;

1. age-associated variation in physiological signs including heart rate, respiratory rate and blood pressure, from birth to adulthood, mean that a single PEWS risk model would be inadequate to flag deterioration across the age ranges [15-18].

2. the ability to physiologically compensate for serious illness is limited in children, providing a shorter window of opportunity for staff or parents to recognise the signs and to respond quickly, to avoid the progression of deterioration to become critical $[19,20]$.

To date, there is no technology similar to that used in adults, which has been robustly tested for use for children in hospital, for screening physiological signs using an underpinning age-specific PEWS risk model. The most extensive randomised study of PEWS in an international context, the EPOCH study, did not demonstrate a reduction in mortality associated with the use of PEWS [1]. A lower observed event rate for mortality occurred in both arms, of the intervention and comparison sites. The observed mortality was lower than the data used for the computation of the sample size estimate (2007 data from fourteen hospitals). The study also highlighted the challenge in achieving complete data capture of the required vital signs which contribute to PEWS when paper-based documentation was used. Less than $5 \%$ of all the recorded data reviewed [1] were complete for recording of the seven components which had contributed to the validation of the bedside PEWS.

The development of electronic physiological surveillance systems suitable for use in a paediatric population is complex and expensive, potentially deterring industry from developing age-specific products. To address this deficit, 
The Learning Clinic (now System C) collaborated with this research team to develop technology underpinned by scientific evidence. Proof-of-concept testing of the VitalPAC Paediatric prototype occurred at this hospital site, alongside existing practice. When compared to paper charts, VitalPAC Paediatric demonstrated improved the accuracy of vital sign documentation; 98.5\% Vs $85.6 \% p<0.02$; PEWS calculation; $94.6 \%$ Vs $55.7 \% p<0.02$ and reduced the documentation time; $68 \mathrm{Vs} 98 \mathrm{~s} p<0.002$ [21].

Sepsis is a major cause of deterioration, therefore addition of a paediatric sepsis [22, 23] clinical prompt, including laboratory and clinical data was tested. This demonstrated $71 \%$ sensitivity and $81 \%$ specificity when two or more components were present, suggesting it could be an effective tool to prompt nurses and doctors to consider sepsis earlier. The staff using the technology perceived that this mode of documentation improved safety and increased team situation awareness.

This study is the whole hospital evaluation of electronic physiological surveillance technology to provide pro-active monitoring for paediatric in-patients.

\section{Methods/design}

SMART technology, self-monitoring and reporting technologies, such as electronic physiological surveillance systems have the potential to provide an end-to-end solution for recognising and responding to serious illness or deterioration in children. The study aims to:

- Standardise the process for monitoring vital signs for children in hospital

- Improve the detection of early signs of clinical deterioration, including sepsis

- Provide real-time data to the entire clinical team to improve team situation awareness about deteriorating patients

- Use clinical decision support to prompt the proactive management of deterioration, and early stabilisation of patient, reducing the progression of deterioration to become critical

- Reduce emergency transfers to critical care following deterioration in hospital

- To prompt timely transfer of deteriorating patients to critical care, who were non-responders to firstline stabilisation interventions

\section{Study design}

This is a non-randomised stepped-wedge prospective mixed methods study exploring the clinical effectiveness, clinical utility and cost-effectiveness of an electronic physiological surveillance system to provide active monitoring of paediatric patients and to screen for early signs of serious illness, deterioration or sepsis.

\section{Population and setting}

The participants will be ward in-patients, aged less than 18 years, at a tertiary children's hospital in the United Kingdom. The study site has 240 in-patient beds, a large emergency department, a 24-bed PICU, a 19-bed HDU and a 4-bed long-term ventilation Unit (LTVU).

The patient profile will include a mix of general paediatric medical and surgical patients and tertiary specialties including oncology, cardiology/cardiac surgery, neurology/neurosurgery, burns, respiratory, renal and orthopaedics. Day-case patients, patients in the neonatal surgical unit or in the PICU will be excluded.

The hospital does not have a Medical Emergency Team or a Critical Care Outreach Team. Patient deterioration is managed by the core clinical team. During the out-of-hours period, patients are managed by the on-call team. If a call to the resuscitation team is made, the responding team comprise of a senior nurse, senior paediatric registrars, resuscitation training officers (daytime only Monday to Friday), the Paediatric Intensive Care registrar and/or an anaesthetist.

\section{The intervention}

The intervention is an electronic physiological surveillance system suitable for use in children in hospital. This is composed of health IT software Careflow Vitals (previously known as VitalPAC) configured with an underpinning age-specific PEWS risk model, which runs wirelessly on Apple hand-held devices. The technology is used to manually document vital signs to screen for early signs of serious illness or deterioration, including sepsis. The software prompts an active monitoring process by standardising the complete documentation of vital signs and clinical assessments, at a frequency linked to the child's PEWS. The completed PEWS are categorised as low, moderate, high or critical risk for deterioration, with associated decision support prompted for each. When a suspicion of sepsis has been documented, a complete sepsis assessment is required to stratify the clinical concern regarding sepsis using the NICE sepsis pathway [22], this prompts targeted care delivery and captures the time critical sepsis bundle compliance.

Careflow Vitals is used in conjunction with Careflow Connect; a secure encrypted communication system for professionals to provide real-time data to the entire clinical team on hand-held devices. Automated alerts about high or critical PEWS categorisation are sent to the Nurse in Charge of the ward and to the responsible clinical team. The chronology of escalation, response and associated actions are captured.

There will be phased implementation of the technology ward by ward, with concurrent assessment of the implementation fidelity. Weekly organisational safety reports will be fed back to wards, clinical teams and managers. 
These will collate reporting of the completeness and frequency of assessment, active management of patients with high or critical PEWS or new sepsis concerns, and the timeliness of emergency transfers to critical care.

\section{Comparison}

Baseline data will be collected prospectively for 1 year preceding the intervention, with patient monitoring of vital signs and sepsis screening recorded in the Electronic Patient Record (Meditech 6). The hospital standard for the monitoring of vital signs for children in hospital, is set out in a clinical policy, together with the expected escalation and response processes. All of the component parts required for the calculation of a core PEWS (respiratory rate, effort of breathing, oxygen saturation ( $\mathrm{SpO} 2)$, oxygen delivery (FiO2), heart rate, capillary refill time and nurse concern) are mandated within the EPR each time vital signs are recorded. Temperature is also recorded but it does not contribute to the PEWS. For new admissions, PEWS score 3 or more or clinical concern, requires that an extended PEWS is undertaken. This incorporates additional vital signs alongside the core PEWS; which include blood pressure, AVPU (rapid neurological assessment of responsiveness; Alert-VerbalPain-Unresponsive) and parental concern. The process for communication for concern of deterioration is through the traditional bleep process.

Following a 3 month implementation period, 1 year of post-intervention data will be collected.

\section{Outcomes}

The primary outcome is unplanned transfers to critical care, either to HDU and/or PICU.

The secondary outcomes are critical deterioration events [24], the patients who had unplanned transfers from in-patient wards to critical care (HDU and/or PICU) and required organ support in the following $12 \mathrm{~h}$. The CDE prevalence per 1000 hospital admissions and per 1000 non-ICU bed days will be reported. The timeliness of critical care transfer will be reported using the Children's Resuscitation Intensity Scale (CRIS) [25]. The severity of illness at unplanned transfer to PICU; the Prognostic index of Mortality (PIM3 [26], the requirement for critical care interventions (mechanical ventilation, non-invasive ventilation, inotropes and/or dialysis), length of critical care stay and outcome. We will report the sepsis screening, suspicion of sepsis, clinical confirmation of 'treat as sepsis', the NICE sepsis bundle compliance and associated outcome.

The activation of the resuscitation team for cardiac arrests, respiratory arrests, or peri-arrest situations in the in-patient wards (excluding PICU) will be reported. All-cause mortality will be reported for the wards included in the study. The overall critical care activity; total admissions, bed days, length of stay, refused admissions (internal/external) and cancellations of planned major surgery due to lack of critical care capacity, will be reported.

\section{Sample size}

The study will collect data from 240 in-patient beds. Based on existing hospital data, over 12 months 300-350 emergency transfers to HDU and/or PICU from inpatient wards would be expected. Previously published work from this centre [8] suggested that a robustly implemented process for proactively recognising and responding to deterioration in hospital, would contribute to a $30 \%$ reduction in total bed days following a CDE, with a median reduction of two critical care bed days per CDE. This is projected to be sufficient for the analysis planned.

The projected $80-100 \%$ bed occupancy in 240 beds is expected to yield a minimum of 6 sets of vital signs/per bed/per day, which is expected to yield a minimum of $630 \mathrm{~K}-780 \mathrm{~K}$ observation sets per year.

\section{Data analysis}

The data analysis is broken down into the four main work-packages in the study.

1. The quantitative analysis of the clinical effectiveness at reducing critical deterioration, will report the prevalence of CDEs per 1000 hospital admissions and per 1000 non PICU bed days to compare the baseline and intervention arm of the study. The predictive ability of the age-specific PEWS risk model, will be reported using the sensitivity, specificity, positive/negative predictive values Modelling of the physiological values will be used to determine evidence-based thresholds for vital signs and logistic regression models will be developed to determine the weighting of components of the PEWS risk model to improve the performance at improving early detection of signs of deterioration. The study will use statistical process control techniques and will explore the feasibility of using stochastic control methods to track the condition of patients over-time to develop a longitudinal component (trend changes in vital signs), within the risk model.

2. The qualitative analysis will focus on the clinical utility of the technology to assist in the recognition and response to deterioration in children in hospital. It will also aim to evaluate the acceptability to children and their families of being monitored in this way. The three core methods which will be used are ethnographically driven observation and 
conversational interviews, short e-surveys, and focused interviews. Qualitative analysis will use an interpretive, reflexive, conceptual thematic analytical approach $[27,28]$ the integration of descriptive codes and themes into robust concepts via Morse's four cognitive analytical processes [29].

3. The analysis of proactive sepsis screening for all patients will describe the prevalence of pre-existing risk factors for sepsis, the cases where sepsis was suspected, the subset where the clinician made a decision to treat as sepsis (blood cultures and intravenous antibiotics), NICE sepsis bundle compliance and associated outcome. Multi-variate modelling will be also be used to determine the components of the sepsis assessment which would contribute to improved discrimination of evolving sepsis.

4. The cost-effectiveness of the technology will be analysed using Health Related Group (HRG) costs per day for the critical care and hospital stay following a CDE for up to 90 days, pre and post intervention. The societal perspective will be used and the time horizon is 1 year. For medical direct costs, patient level hospital costs will be computed by using hospital costs and published tariffs where appropriate. Indirect costs include work absenteeism in the parents of children who had an in-hospital deterioration, and productivity losses resulting from the sequelae of critical illness.

\section{Discussion}

The type of active monitoring has been in place for adults in hospital for more than 10 years [12-14]. It was evaluated in two large university hospitals in an NHS setting in the UK and was associated with a reduction in in-hospital mortality [11]. The ability to record real-time vital signs data on mobile devices which are instantly visible to the entire clinical team, together with automated critical alerts, tracked monitoring, escalation and response provides metrics for hospitals to review their patient safety at ward, clinical teams and organisational levels. Although mortality is much rarer for children in hospital, it is envisaged that the use of active monitoring could reduce the progression of deterioration to become critical.

Critical Deterioration Events [30] were associated with a 13-fold increased risk of mortality in hospital. It is a clearly defined outcome measure for deterioration which has been reported in two paediatric studies [1, 30]. There is widespread acceptance that the threshold for admission to critical care varies world-wide, therefore, it is useful to subdivide the admissions to be explicit about CDEs, although, at present, this categorization is not routinely undertaken. However, there is evidence about unplanned admissions to PICU that does demonstrate a reduction in the median length of critical care stay by 2 days following the implementation of a paper-based PEWS [8].

Despite this, it has been challenging to sustain those improvements, over time, not least because of the human resource required to ensure that organisational process are followed. The use of self-monitoring and reporting technologies (SMART) can provide frequent feedback to healthcare professionals and managers to drive culture change, and can provide assurance to healthcare regulators of safe processes for patients. Up to now paper-based monitoring of vital signs documentation and organisational processes has meant that it is difficult to ensure adequate implementation fidelity of PEWS.

If the technology is clinically effective at reducing CDE in hospitalised children, it could be deployed widely, to reduce the morbidity and mortality associated with critical deterioration.

\section{Abbreviations}

CDE: Critical Deterioration Events; CRIS: Children's Resuscitation Intensity Scale; HDU: High Dependency Unit; NiHR: National Institute for Health Research; PICU: Paediatric Intensive Care Unit; PIM3: Paediatric Index of Mortality, version 3

\footnotetext{
Acknowledgements

This report is independent research funded by the National Institute for Health Research (Invention for Innovation programme (i4i), Active monitoring of paediatric in-patients using "VitalPac Paediatric" to reduce emergency transfer to Critical Care following deterioration in hospital, II-LA0216-20002. The views expressed in this publication are those of the author(s) and not necessarily those of the National Institute for Health Research or the Department of Health and Social Care.

Mrs. Hilda Gwilliams, Chief Nurse, Alder Hey Children's NHS Foundation Trust and executive sponsor for the DETECT study, who provided vision, leadership and unwavering support for improving health outcomes for children in hospital.

Mrs. Kate Warriner, Chief Digital and Information Officer, Alder Hey Children's NHS Foundation Trust, who provided leadership within the digital strategy to support this research study.

Dr. James Bunn; WHO Lead for Child Health, Sierra Leone, who contributed to developing this research idea and writing the funded research application, while employed at Alder Hey.

Mr. Markus Bolton, CEO System C, who agreed to continue as industry partner to the study, once System C took over The Learning Clinic.
}

\section{Authors' contributions}

GS; Co-Chief Investigator. Concept and design of the study, Obtained funding for the study, Lead author for the manuscript. Corresponding author gerri.sefton@alderhey.nhs.uk. BC; Professor of Children's Nursing, co-applicant. Lead for qualitative research within the study. Contributed to drafting the research protocol and the manuscript. SL; Medical Statistician, co-applicant. Lead for statistical analyses for the study. Contributed to drafting the research protocol and the manuscript. MP; Co-Director of NIHR Alder Hey Clinical Research Facility, co-applicant. Obtained funding for the study, Contributed to drafting the research protocol and the manuscript. CM; Senior lecturer in Health Economics, Contributed to drafting the research protocol and the manuscript. JP; Senior Patient and Public Involvement Manager, NIHR Alder Hey Clinical Research Facility, Contributed to drafting the research protocol and the manuscript. Approved the final manuscript. FM; Consultant Paediatrician, co-applicant. Contributed to drafting the research protocol and the manuscript. BH; Professor of health Economics University of Lancaster. Co-applicant, Contributed to drafting the research protocol and the manuscript. RK; Roger Killen, Previous CEO of The Learning Clinic, Co-applicant. Obtained funding for the study, Contributed to drafting the research protocol and the manuscript. EDC; Co-Chief Investigator. Concept and design of the study, Academic lead for the 
study, Obtained funding for the study, Contributed to drafting the manuscript. All authors read and approved the final manuscript.

\section{Funding}

The study protocol presented in this study was peer reviewed by the funding body, the National Institute for Health Research (NIHR) and funding was awarded.

System $C$, the industry partner, was required to provide matched funding for the development work for Careflow Vitals use in paediatrics. The research study proposal was developed by clinicians and was not influenced by the industry partner. The role of the industry partner was to advise on the technical feasibility of developing the technology to deliver the study. The study design incorporates work packages to evaluate the clinical utility of the technology and to evaluate the cost-effectiveness of the technology as required for research applications submitted under the i4i funding scheme. The draft of the manuscript for publication was reviewed by the Study Management Group, comprising of clinicians with expertise in paediatrics, the management of deterioration in children in hospital and in patient safety.

\section{Availability of data and materials}

A minimum dataset associated with the study outcomes, will be made available through linked documents uploaded alongside any future publications.

\section{Ethics approval and consent to participate}

Ethics approval was given for the study, by the regional ethics committee, REC 17/NW/0533, and the Health Research Authority (HRA) permission to proceed was issued, IRAS ID 215339. The study was also submitted to the Medicines and Healthcare products Regulatory Agency (MHRA) which confirmed that it did not fall within its trial regulations.

Vital signs documentation and sepsis screening are part of routine care and consent is not required when the device is used to record these changes. However, posters outlining the purpose of the study will be displayed in the in-patient wards, giving permission to parents to decline the use of their child's anonymised vital signs data in the study analysis.

Consent will be collected for the families of children who are transferred to critical care following deterioration, so that additional data can be captured about the clinical course, and to ascertain the indirect financial cost to families associated with critical deterioration. Consent will also be sought from a selection of patients who did and did not deteriorate to explore the acceptability of being monitored using this technology. The consent process incorporates statements about the use of anonymised data for publication. Consent will also be sought from the staff who agree to participate in the evaluation of clinical utility of the devices.

\section{Consent for publication}

Consent for publication is incorporated within the consent form used to document consent for participants, and their families. This explicitly states that no patient identifiable data will be published.

\section{Competing interests}

The authors declare that they have no competing interests.

\section{Author details}

${ }^{1}$ Alder Hey Children's NHS Foundation Trust, Liverpool L12 2AP, UK. ²Edge Hill University, Ormskirk L39 4QP, UK. ${ }^{3}$ University of Liverpool, Liverpool L69 3BX, UK. ${ }^{4} \mathrm{NIHR}$ Alder Hey Clinical Research Facility, Alder Hey Children's NHS Foundation Trust, Liverpool L12 2AP, UK. ${ }^{5}$ Lancaster University , Lancashire LA1 4YG, UK. ${ }^{6}$ University of Exeter, Exeter EX4 4PU, UK.

\section{Received: 29 July 2019 Accepted: 24 September 2019}

\section{Published online: 17 October 2019}

\section{References}

1. Parshuram CS, Dryden-Palmer K, Farrell C, Gottesman R, Gray M, Hutchison JS, Helfaer M, Hunt EA, Joffe AR, Lacroix J. Effect of a pediatric early warning system on all-cause mortality in hospitalized pediatric patients: the EPOCH randomized clinical trial. JAMA. 2018;319(10):1002-12.

2. Confidential Enquiry into Maternal \& Child Health deaths. In: Pearson G, editor. Why Children Die; A pilot study. London: CEMACH; 2008.
3. Roland D, Oliver A, Edwards ED, Mason BW, Powell CV. Use of paediatric early warning systems in Great Britain: has there been a change of practice in the last 7 years? Arch Dis Child. 2014;99(1):26-9.

4. PICANet: Paediatric Intensive Care Audit Network Annual Report. Tables and Figures; Data collection period January 2015-December 2017. Published 2018.

5. Odetola F, Clark S, Dechert R, Shanley T. Going back for more: an evaluation of clinical outcomes and characteristics of readmissions to a pediatric intensive care unit. Pediatr Crit Care Med. 2007;8(4):343-7.

6. Odetola FO, Rosenberg AL, Davis MM, Clark SJ, Dechert RE, Shanley TP. Do outcomes vary according to the source of admission to the pediatric intensive care unit? Pediatr Crit Care Med. 2008;9(1):20-5.

7. Kotsakis A, Stevens D, Frndova H, Neal R, Williamson G, Mohseni-Bod H, Parshuram CS. Description of PICU Unplanned Readmission. Pediatr Crit Care Med. 2016;17(6):558-62

8. Sefton G, McGrath C, Tume L, Lane S, Lisboa P, Carrol E. What impact did a Paediatric Early Warning system have on emergency admissions to the paediatric intensive care unit? An observational cohort study. Intensive Crit Care Nurs. 2015;31(2):91-9.

9. Chapman SM, Wray J, Oulton K, Pagel C, Ray S. Peters MJ: 'The Score Matters': wide variations in predictive performance of 18 paediatric track and trigger systems. Arch Dis Child. 2017;102(6): 487-95.

10. Smith GB, Prytherch DR, Schmidt P, Featherstone PI, Knight D, Clements G, Mohammed MA. Hospital-wide physiological surveillance-a new approach to the early identification and management of the sick patient. Resuscitation. 2006;71(1):19-28.

11. Schmidt PE, Meredith P, Prytherch DR, Watson D, Watson V, Killen RM Greengross P, Mohammed MA, Smith GB. Impact of introducing an electronic physiological surveillance system on hospital mortality. BMJ Qual Saf. 2015;24(1):10-20.

12. Prytherch DR, Smith GB, Schmidt PE, Featherstone PI. ViEWS--Towards a national early warning score for detecting adult inpatient deterioration. Resuscitation. 2010;81(8):932-7.

13. Royal College of Physicians. National Early Warning Score (NEWS) : Standardising the assessment of acute-illness severity in the NHS. London: RCP; 2012.

14. Royal College of Physicians. National Early Warning Score (NEWS 2); Standardising the assessment of acute-illness severity in the NHS. London: RCP; 2017.

15. Fleming $S$, Thompson M, Stevens R, Heneghan C, Plüddemann A Maconochie I, Tarassenko L, Mant D. Normal ranges of heart rate and respiratory rate in children from birth to 18 years of age: a systematic review of observational studies. The Lancet. 2011;377(9770):1011-8.

16. O'Leary F, Hayen A, Lockie F, Peat J. Defining normal ranges and centiles for heart and respiratory rates in infants and children: a cross-sectional study of patients attending an Australian tertiary hospital paediatric emergency department. Arch Dis Child. 2015;100(8):733-7.

17. Bonafide C, Brady P, Keren R, Conway P, Marsolo K, Daymont C. Development of heart and respiratory rate percentile curves for hospitalised children. Pediatrics. 2013;131(4):1150-7.

18. Jackson LV, Thalange NK, Cole TJ. Blood pressure centiles for Great Britain. Arch Dis Child. 2007;92(4):298-303.

19. Convertino VA, Wirt MD, Glenn JF, Lein BC. The compensatory reserve for early and accurate prediction of hemodynamic compromise: a review of the underlying physiology. Shock. 2016;45(6):580-90.

20. Aneja RK, Carcillo JA. Differences between adult and pediatric septic shock. Minerva Anestesiol. 2011;77:986-92.

21. Sefton G, Lane S, Killen R, et al. Accuracy and efficiency of recording Paediatric Early Warning Scores (PEWS) using electronic physiological surveillance systems compared with traditional paper-based charting. Comput Inform Nurs. 2017;35(5):228.

22. National Institute for Health and Care Excellence, NICE. Sepsis: recognition, diagnosis and early management (NG51). London: NICE; 2016.

23. Freitag A, Constanti M, O' Flynn N, Faust SN. Suspected sepsis: summary of NHS guidance. BMJ. 2016;354:14030.

24. Bonafide $C P$, Roberts KE, Priestly MA. Development of a pragmatic measure for evaluating and optimizing rapid response systems. Arch Pediatr. 2012; 129(4):e874-81.

25. Parshuram CS, Bayliss A, Reimer J, Middaugh K, Blanchard N. Implementing the Bedside Paediatric Early Warning System in a community hospital: A prospective observational study. Paediatr Child Health. 2011;16(3):e18-22. 
26. Tyagi P, Tullu MS, Agrawal M. Comparison of Pediatric Risk of Mortality III, Pediatric Index of Mortality 2, and Pediatric Index of Mortality 3 in

Predicting Mortality in a Pediatric Intensive Care Unit. J Pediatr Intensive Care. 2018;7(4):201-6.

27. Thorne S. Interpretive Description. Walnut Creek: Left Coast Press; 2016.

28. Braun V, Clarke V. Using thematic analysis in psychology. Qualitative Res Psychol. 2006;3:25.

29. Morse JM. Emerging from the data: Cognitive processes of analysis in qualitative inquiry. In: Morse J, editor. Critical Issues in Qualitative Research. Menlo Park: Sage; 1994. p. 23-43.

30. Bonafide CP, Localio AR, Roberts KE, Nadkarni VM, Weirich CM, Keren R. Impact of rapid response system implementation on critical deterioration events in children. JAMA Pediatr. 2014;168(1):25-33.

\section{Publisher's Note}

Springer Nature remains neutral with regard to jurisdictional claims in published maps and institutional affiliations.

- fast, convenient online submission

- thorough peer review by experienced researchers in your field

- rapid publication on acceptance

- support for research data, including large and complex data types

- gold Open Access which fosters wider collaboration and increased citations

- maximum visibility for your research: over $100 \mathrm{M}$ website views per year

At $\mathrm{BMC}$, research is always in progress. 